Case Study

\title{
Study of magnet fall through conducting pipes using a data logger
}

\author{
Abdul Kareem Thottoli ${ }^{1}$ - Mohammed Fayis $^{1} \cdot$ T. C. Mohamed $^{1} \cdot$ T. Amjad $^{1} \cdot$ P. T. Shameem ${ }^{1} \cdot$ Muhammed Mishab $^{1}$
}

(c) Springer Nature Switzerland AG 2019

\begin{abstract}
Amusing students by dropping a magnet through a metal pipe is a common activity in many classrooms. Students will be excited to see the slow movement of the magnet through the pipe due to the eddy current and its damping force. This activity can be performed to enhance their knowledge about the properties of magnet and metal pipes if we are able to get data from this experiment. This case study prepared by performing the magnet fall activity by incorporating ExpEYES17 data logger and extracted the information such as the magnetic flux, terminal velocity of the magnet in the pipe, dragging coefficient and conductivity of the metal pipe.
\end{abstract}

Keywords Conducting pipe · Dragg coefficient · Eddy current $\cdot$ ExpEYES $\cdot$ Magnet fall

\section{Introduction}

Magnet falling through a conducting pipe is generally demonstrated in almost every classroom to amuse the students about the effect of eddy current. Here an experiment is described to analyze the damping fall of a magnet through a metallic pipe using the ExpEYES17 data logger. The fall of a magnet inside a conducting pipe is damped by the opposing force due to the eddy current generated in the conducting pipe. This phenomenon is demonstrated in many places and many people have described the mathematical model of the fall of the magnet through the conducting pipe [1-12]. Further, this magnetic damping is exploited in a diversity of important applications including advanced car suspension systems that use a colloidal suspension, the smooth braking. Here we are presenting a case study by verifying the theoretical models by finding the drag coefficient and the conductivity of a conducting pipe using the previously derived equations.

\section{Theory}

The basic theory is explained in many papers [1-12], though a short description is given here for understanding the problem. When a permanent magnet is released through a metallic pipe, the magnetic flux through the metallic surface changes as it falls. According to Faraday's Law, the flux change induces an electromotive force and then an electric current through the surface of the metallic pipe. This electric current produces a magnetic field to oppose the action that induced the emf. This repulsion yields a damping force on the magnet, so the magnet drifts through the pipe. The action of the forces on the magnet is shown in Fig. 1. A generally accepted model of velocity-dependent resistive forces can be written as Eq. (1) [13].

$\mathrm{F}=\mathrm{k}_{1} \mathrm{v}+\mathrm{k}_{2} \mathrm{v}^{2}$

In our case, the velocity is assumed to be small, therefore the term ' $\mathrm{v}^{21}$ will be negligible and it is ignored here. Further, the air resistance is also neglected. Therefore, two forces are acting on the magnet, one is the gravity which pulls the magnet down and the other is the

Abdul Kareem Thottoli, abdulkareem.t@gmail.com | ${ }^{1}$ Department of Physics, Pocker Sahib Memorial Orphanage College (PSMO College), Tirurangadi, Malappuram, Kerala 676306, India. 

the falling magnet in a metallic pipe
Fig. 1 Free body diagram of

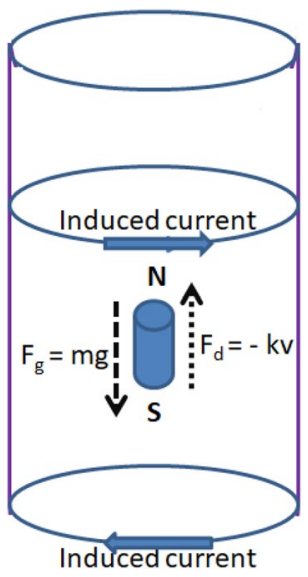

induced magnetic force which tries to slow down the falling magnet.

The net force on the magnet can be calculated by summing the downward force and the dragging force. When ' $m$ ' and ' $v$ ' are the mass and velocity of the magnet respectively, the governing equation of motion can be written as;

$\mathrm{m} \frac{\mathrm{d} v}{\mathrm{dt}}=\mathrm{mg}-\mathrm{kv}$

where ' $g$ ' is the acceleration due to gravity (value of $g$ is $9.8 \mathrm{~m} \mathrm{~s}^{-2}$ ) and ' $k$ ' is the damping coefficient. When ' $v_{0}$ ' is the initial velocity then the velocity will be found by integrating Eq. (2) as;

$v(t)=\left(v_{0}-\frac{m g}{k}\right) e^{-\frac{k}{m} t}+\frac{m g}{k}=\frac{d x(t)}{d t}$

Then integrate the Eq. (3) to get $x(t)$,

$x(t)=\left(\frac{k v_{0} m-m^{2} g}{k^{2}}\right)-\left(\frac{k v_{0} m-m^{2} g}{k^{2}}\right) e^{-\left(\frac{k}{m} t\right)}+\frac{m g}{k} t$

By rearranging the equation, we get

$\frac{x}{m^{2} g} k^{2}-\left(\frac{v_{0}}{m g}+\frac{t}{m}\right) k+1=\left(1-\frac{v_{0} k}{m g}\right) e^{-\left(\frac{t}{m}\right) k}$

Damping coefficient, ' $\mathrm{k}$ ' can be found by solving the above Eq. (5) by substituting the corresponding values of the distance ' $x$ ' (length of the pipe), mass of the magnet ' $m$ ', initial velocity ' $v_{0}$ ', the value of ' $g$ ' and the time taken by the magnet to pass through the pipe ' $t$ '. The time ' $t$ ' to pass the length of the pipe will be obtained using ExpEYES17 data logger by connecting two coils fixed at each end of the pipe (for details http://expeyes.in). Equation (5) solved by using www.wolframalpha.com to find $\mathrm{k}$ values.

For finding the conductivity of metallic pipe, Iniguez et al. [7] developed an equation by connecting the conductivity and speed of the falling object as given below,

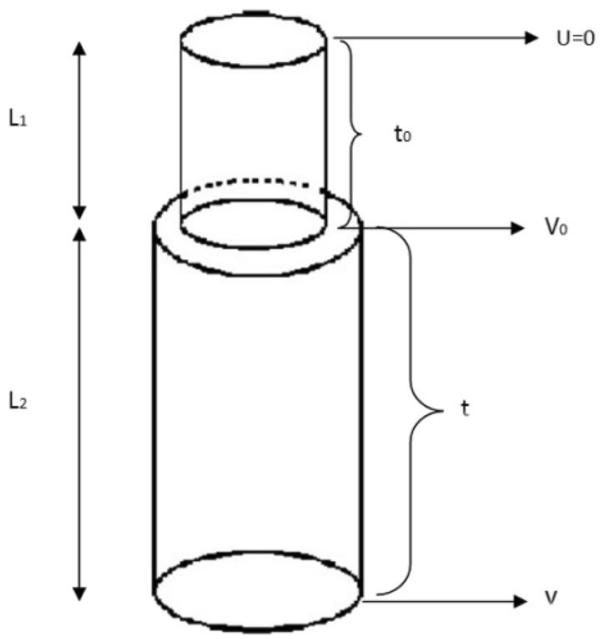

Fig. 2 Description of time and velocity

$\sigma=\frac{\sqrt{2} \pi^{2} k}{2 \varphi_{0}^{2}} \sqrt{\frac{c^{2}-b^{2}}{\ln ^{3}\left(\frac{c}{b}\right)}}$

where ' $b$ ' and ' $c$ ' are the inner and the outer radius of the pipe respectively, and ' $k$ ' is the damping coefficient. Where ' $\varphi_{0}$ ' is the maximum value of magnetic flux ' $\varphi$ ', and will be obtained by numerically integrating (trapezoidal rule) the Eq. (7) using Microsoft Excel sheet. Expeyes 17 was used to collect the emf versus time data.

$\epsilon=-\frac{\mathrm{d} \varphi}{\mathrm{dt}}$

The initial velocity of the magnet was found by the time of flight of the magnet through the PVC of length $8.4 \mathrm{~cm}$ fixed above the experimenting pipes. The whole idea is depicted in the Fig. 2. The time of flight can be calculated theoretically using the Eq. (8) and experimental time was found by fixing two coils at each end of the pipe as given in the Fig. 3,

$t_{0}=\sqrt{\frac{2 L_{1}}{g}}$

Then the initial velocity of the magnet when the magnet entering the conductive pipe will be

$\mathrm{V}_{0}=\mathrm{gt}_{0}$

The effect of air in the downward motion of the magnet when it is passing through a pipe was found by dropping the test magnet through a non-conducting PVC pipe of known length. Then the theoretical time of flight through 
Fig. 3 Schematic diagram of the experimental setup

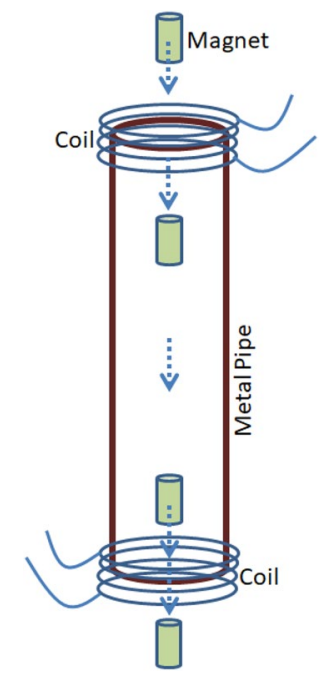

Table 1 Characteristics of the pipes

\begin{tabular}{lllll}
\hline Type & Length $(\mathrm{cm})$ & $\begin{array}{l}\text { Inner } \\
\text { diameter } \\
(\mathrm{cm})\end{array}$ & $\begin{array}{l}\text { Outer } \\
\text { diameter } \\
(\mathrm{cm})\end{array}$ & Thickness (cm) \\
\hline $\mathrm{Cu}(\mathrm{P} 1)$ & 25.12 & 1.393 & 1.601 & 0.104 \\
$\mathrm{Cu}(\mathrm{P} 2)$ & 25.3 & 1.592 & 1.938 & 0.173 \\
Brass (P3) & 25.36 & 1.6885 & 1.9185 & 0.115 \\
Brass (P4) & 25.2 & 1.4025 & 1.595 & 0.09625 \\
SS (P5) & 25.06 & 1.78 & 1.981 & 0.1005 \\
SS (P6) & 25.18 & 1.3995 & 1.637 & 0.11875 \\
Al (P7) & 25.26 & 1.7375 & 1.955 & 0.10875 \\
\hline
\end{tabular}

the larger PVC, $25.3 \mathrm{~cm}$ long, can be determined by solving the quadratic equation

$\mathrm{L}_{2}=v \mathrm{t}+\frac{1}{2} g \mathrm{t}^{2}$

\section{Experimental setup}

Damping coefficient was found by solving the Eq. (5) after substituting the corresponding values of ' $x$,' $m m^{\prime},{ }^{\prime}{ }^{\prime}, v_{0}$ ' and ' $t$ '. For our experiments, we had used fixed length of metallic pipes (as obtained from market) and magnets of known weights as given in the Tables 1 and 2 . So, finding the time covered by the test magnet to cross the metallic pipes helps us to find the damping coefficient in that pipe. Recording of time in these small pipes are tedious and difficult using ordinary stopwatches and other conventional methods. Accurate time recording is only possible by sensors controlled by microcontrollers. So, here a computer interfaced data acquisition tool ExpEYES17 was used to record accurate time along with a coil as a sensor.
Table 2 Characteristics of the magnets

\begin{tabular}{lclll}
\hline Magnets & Mass $(\mathrm{g})$ & Diameter $(\mathrm{cm})$ & Length $(\mathrm{cm})$ & $\begin{array}{l}\text { Magnetic } \\
\text { moment } \\
\left(\mathrm{A} \mathrm{m}^{2}\right)\end{array}$ \\
\hline Magnet A & 9.844 & 1.204 & 1.21 & 9.8713 \\
Magnet B & 14.842 & 1.204 & 1.804 & 14.986 \\
Magnet C & 19.78 & 1.204 & 2.414 & 20.139 \\
Magnet D & 24.768 & 1.204 & 3.004 & 26.0028
\end{tabular}

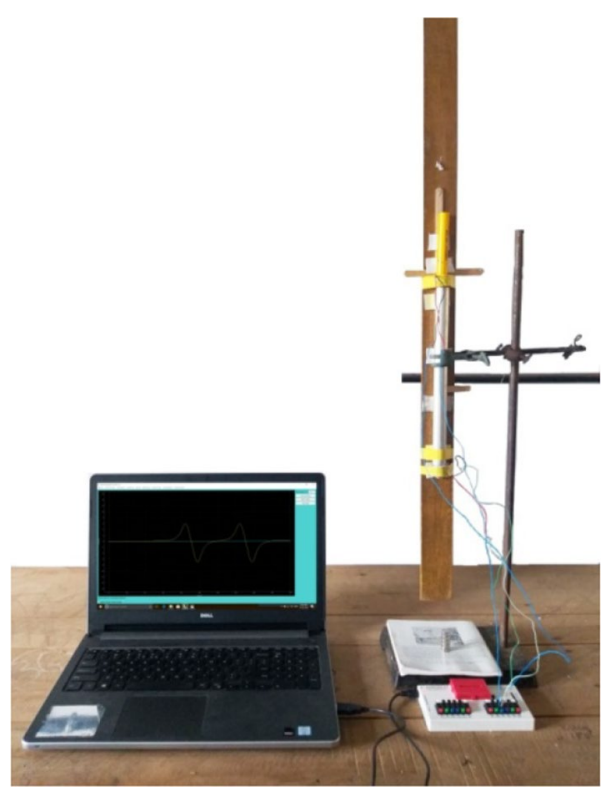

Fig. 4 Photograph of the experimental setup

ExpEYES17 is a hardware and software framework for realtime measurement and data analysis. The sensor used in our experiment was a coil that senses the change of magnetic flux. The sensor coils were fixed at each end of the metallic pipe and on PVC, as the center of the coil touches exactly at the ends of the pipes. Then the coil wires were connected to the ExpEYES17 as given in the Induction Coil experiments section of ExpEYES17 software. Schematically the experimental setup is shown in Fig. 3 and the photograph of the experimental setup with pipes and magnets are shown in Figs. 4 and 5.

Magnets of different weights and sizes were dropped through a plastic non-conducting pipe from $8.4 \mathrm{~cm}$ above from the end of the metallic pipes as shown in Fig. 3 by keeping the ExpEYES17 data logger in ON state. When the magnet passes through the coils fixed at each end of the metallic pipes, the time changing flux induces some emf. This emf versus time data were recorded using the ExpEYES17 data logger option. ExpEYES17 software has an option to plot this recorded data, however we have 


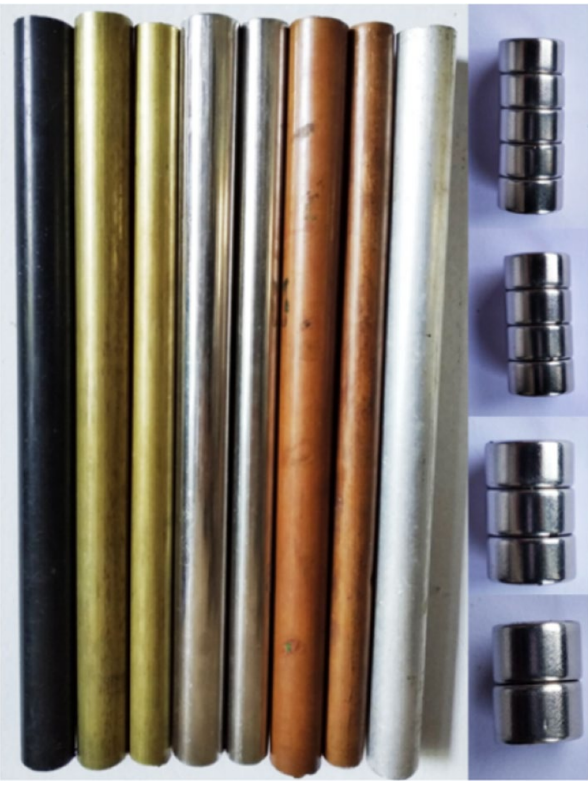

Fig. 5 Pipes and magnets

used Gnuplot software for finding exact time in between the zero crossing of the emf versus time plots generated by the two coils. The zero crossing or the midpoint of the resultant plots shows that the magnet reached the ends of the metallic pipe. For this experiment, pipes and magnets of different sizes and weights were used and their diameters were measured using a screw gauge. The measured values for different pipes are given in Table 1. Magnets length and mass are given in Table 2. Magnetic moments were found using deflection magneto meter setup and tabled in Table 2.

\section{Results and discussion}

The initial velocity of the magnet was found by fixing two coils at each end of the PVC pipe of length $8.4 \mathrm{~cm}$. Observed time and initial velocities are given in Table 3. The time values were substituted in Eq. (9) for finding initial velocity ' $v_{0}$ '. Before performing the experiment with

Table 3 The initial velocity of the magnet

\begin{tabular}{lll}
\hline Magnets & $\begin{array}{l}\text { Time to cover } 8.4 \mathrm{~cm} \\
\text { from emf plot (in s) }\end{array}$ & $\begin{array}{l}\text { Velocity of the magnet when } \\
\text { entering to metal pipe } \mathrm{v}_{0}=\mathrm{gt} \\
\left(\mathrm{m} \mathrm{s}^{-1}\right)\end{array}$ \\
\hline Magnet A & 0.147575 & 1.446235 \\
Magnet B & 0.140674 & 1.378605 \\
Magnet C & 0.143248 & 1.403830 \\
Magnet D & 0.135928 & 1.332097 \\
\hline
\end{tabular}

conducting pipes, we found the time to pass the magnets through a non-conducting pipe (here PVC) of length $25.3 \mathrm{~cm}$ and $2.5 \mathrm{~cm}$ of internal diameter. The obtained time values from ExpEYES17 data were compared with theoretical time. Theoretical time was found out as $0.1313 \mathrm{~s}$ by using Eq. (8). The time data shows that there is a difference in theoretical and experimental time that may be due to the air drag in pipes.

Theoretical and experimental time for PVC for the test magnets are given in Table 4. The calculated data given in Table 4 shows that the magnet feels some upward thrust due to the air present in the PVC pipe. From this information, we deduce that the test magnet feels both the magnetic drag and the air resistance when the magnet moves through metallic pipe. The experiment was repeated for different conducting pipes and the results are tabulated in Table 5.

The data is also plotted in the Fig. 6 with the moment per mass of magnet versus drag coefficient. The plot shows that the copper has the highest drag coefficient than the other tested materials. When comparing the ' $k$ ' values of copper, the plot shows that the ' $k$ ' value increases with the thickness of the pipe as well as the strength of the magnet. But, in the case of SS, the magnetic drag force is not considerable and it approached the air drag as seen in PVC when the mass of the magnet increased. This plot also shows that the arrangement of materials based on their electrical conductivity in decreasing order from top to bottom.

Here in our case, the strength of the magnet is also increasing with increasing the mass. From the Fig. 6 , it is clear that when the strength of the magnet increases the damping coefficient ${ }^{\prime} k$ ' increases exponentially and tending to a constant value in all the pipes we used except in the case of stainless steel pipe. From the data, it is deduced that the drag coefficient is not a constant value for a particular material, but it depends on many other

Table 4 Initial velocity, time and drag coefficient of PVC (theoretical and experimental)

\begin{tabular}{llll}
\hline PVC pipe (25.3 cm) & Time (in s) & $\begin{array}{l}\text { Initial velocity } \\
\left(\mathrm{m} \mathrm{s}^{-1}\right)\end{array}$ & $\begin{array}{l}\text { Drag coef- } \\
\text { ficient k } \\
\left(\mathrm{kg} \mathrm{s}^{-1}\right)\end{array}$ \\
\hline Theoretical & 0.13132 & 1.283121 & 0 \\
& 0.13132 & 1.283121 & 0 \\
& 0.13132 & 1.283121 & 0 \\
Experimental & 0.13132 & 1.283121 & 0 \\
& 0.18365 & 1.446235 & 0.074389 \\
& 0.17228 & 1.378605 & 0.090034 \\
& 0.16645 & 1.403830 & 0.111809 \\
& 0.162471 & 1.332097 & 0.116745 \\
\hline
\end{tabular}


Table 5 Drag coefficient $k$ of different pipes

\begin{tabular}{|c|c|c|c|c|}
\hline Pipe & Length $(\mathrm{cm})$ & Time (in s) & $\begin{array}{l}\text { Drag coeffi- } \\
\text { cient k }\left(\mathrm{kg} \mathrm{s}^{-1}\right)\end{array}$ & $\begin{array}{l}\text { Terminal } \\
\text { velocity } \\
\left(\mathrm{m} \mathrm{s}^{-1}\right)\end{array}$ \\
\hline \multirow[t]{4}{*}{ P1 } & 25.12 & 1.3237 & 0.558259 & 0.1721 \\
\hline & & 1.0454 & 0.674019 & 0.2221 \\
\hline & & 0.8439 & 0.74116 & 0.2832 \\
\hline & & 0.6769 & 0.753654 & 0.3332 \\
\hline \multirow[t]{4}{*}{ P2 } & 25.3 & 1.8062 & 0.739919 & 0.1332 \\
\hline & & 1.6033 & 0.99404 & 0.1443 \\
\hline & & 1.3122 & 1.10138 & 0.1721 \\
\hline & & 1.1217 & 1.18653 & 0.1999 \\
\hline \multirow[t]{4}{*}{ P3 } & 25.36 & 0.5068 & 0.232846 & 0.4186 \\
\hline & & 0.5517 & 0.374371 & 0.3943 \\
\hline & & 0.4491 & 0.416463 & 0.4672 \\
\hline & & 0.3427 & 0.398303 & 0.6130 \\
\hline \multirow[t]{4}{*}{ P4 } & 25.2 & 0.5210 & 0.240269 & 0.3992 \\
\hline & & 0.4555 & 0.317085 & 0.4624 \\
\hline & & 0.3501 & 0.333727 & 0.5887 \\
\hline & & 0.2899 & 0.338539 & 0.7199 \\
\hline \multirow[t]{4}{*}{ P5 } & 25.06 & 0.2473 & 0.118902 & 0.8152 \\
\hline & & 0.2674 & 0.190438 & 0.7666 \\
\hline & & 0.2441 & 0.230728 & 0.8429 \\
\hline & & 0.2258 & 0.251202 & 0.9679 \\
\hline \multirow[t]{4}{*}{ P6 } & 25.18 & 0.3507 & 0.168425 & 0.5687 \\
\hline & & 0.2692 & 0.19065 & 0.7626 \\
\hline & & 0.2304 & 0.212866 & 0.9123 \\
\hline & & 0.1694 & 0.136985 & No drag \\
\hline \multirow[t]{4}{*}{ P7 } & 25.26 & 0.7248 & 0.321479 & 0.3020 \\
\hline & & 0.6151 & 0.414589 & 0.3555 \\
\hline & & 0.5272 & 0.483126 & 0.4089 \\
\hline & & 0.4396 & 0.506083 & 0.4818 \\
\hline \multirow[t]{4}{*}{ PVC } & 25.3 & 0.1836 & 0.074389 & No drag \\
\hline & & 0.1722 & 0.090034 & No drag \\
\hline & & 0.1664 & 0.111809 & No drag \\
\hline & & 0.1624 & 0.116745 & No drag \\
\hline
\end{tabular}

quantities such as magnetic moment, conductivity of the material, inner and outer diameter of the pipe.

Terminal velocity of the magnets when it moves in a conducting pipe can also be found by these experiments. Terminal velocity was found by plotting velocity versus time data. Velocity versus time plot for $\mathrm{Cu}$ pipe (P1) is given in Fig. 7, other plots have not shown here to save space but the extracted data is given in Table 5 .
It is seen that the magnets attains the terminal velocity within $0.1 \mathrm{~s}$ in highly conducting pipes than the other. Further, from the plots we can say that the terminal velocity is almost independent of the magnet weight in the case of higher conducting pipes.

Further it is observed from the velocity-time plot of different conducting pipes, that when the magnet falls into the pipe, their velocity is exponentially decreases by the action of opposing force due to eddy current and it attains the terminal velocity very quickly. It is clear that the time taken for attaining the terminal velocity is dependent on the conductivity of the material of the pipe.

The conductivity of the pipes is calculated using the Eq. (6) and tabulated in Table 7. For finding the conductivity using the equation, maximum flux $\left(\varphi_{0}\right)$ is required. Therefore, maximum flux $\left(\varphi_{0}\right)$ was found by integrating the induced emf values using trapezoidal rule. The induced emf and the integrated values were plotted for all magnets and found the $\varphi_{0}$ value. Plots of two and four magnets are only shown in Fig. 8. Extracted values of maximum flux for each magnet from their corresponding plots are given in Table 6.

Table 7 shows the conductivity of the materials of the pipe by magnet damped fall method and they are compared with standard values. The calculated values are almost matching with the standard value of the tested materials. Though, it is understood from this experiment that this is not an efficient method to find the conductivity of materials.

\section{Conclusions}

Experiments have been performed to test the previously reported theoretical model of the retarded fall of a cylindrical magnet inside a vertical conductive pipe and the results are discussed here. We have used previously derived equations to find the damping coefficient ' $k$ ' during the retarded fall of the magnet through a conducting pipe. Further, the conductivity of seven different pipes was also calculated using ' $k$ ' values and maximum flux through the pipes. From this experiment, we observed that the drag force depends on the conductivity of the pipe, its inner and outer radii and the magnetic moment of the magnet used. Further, it is seen that the velocity of the magnet exponentially decreases and attains the terminal velocity within seconds in electrically conducting pipes. 
Fig. 6 Variation of damping coefficient $\mathrm{k}$ with the ratio of the moment to the mass of the magnets

Fig. 7 Velocity versus time plot for Cu pipe (P1)
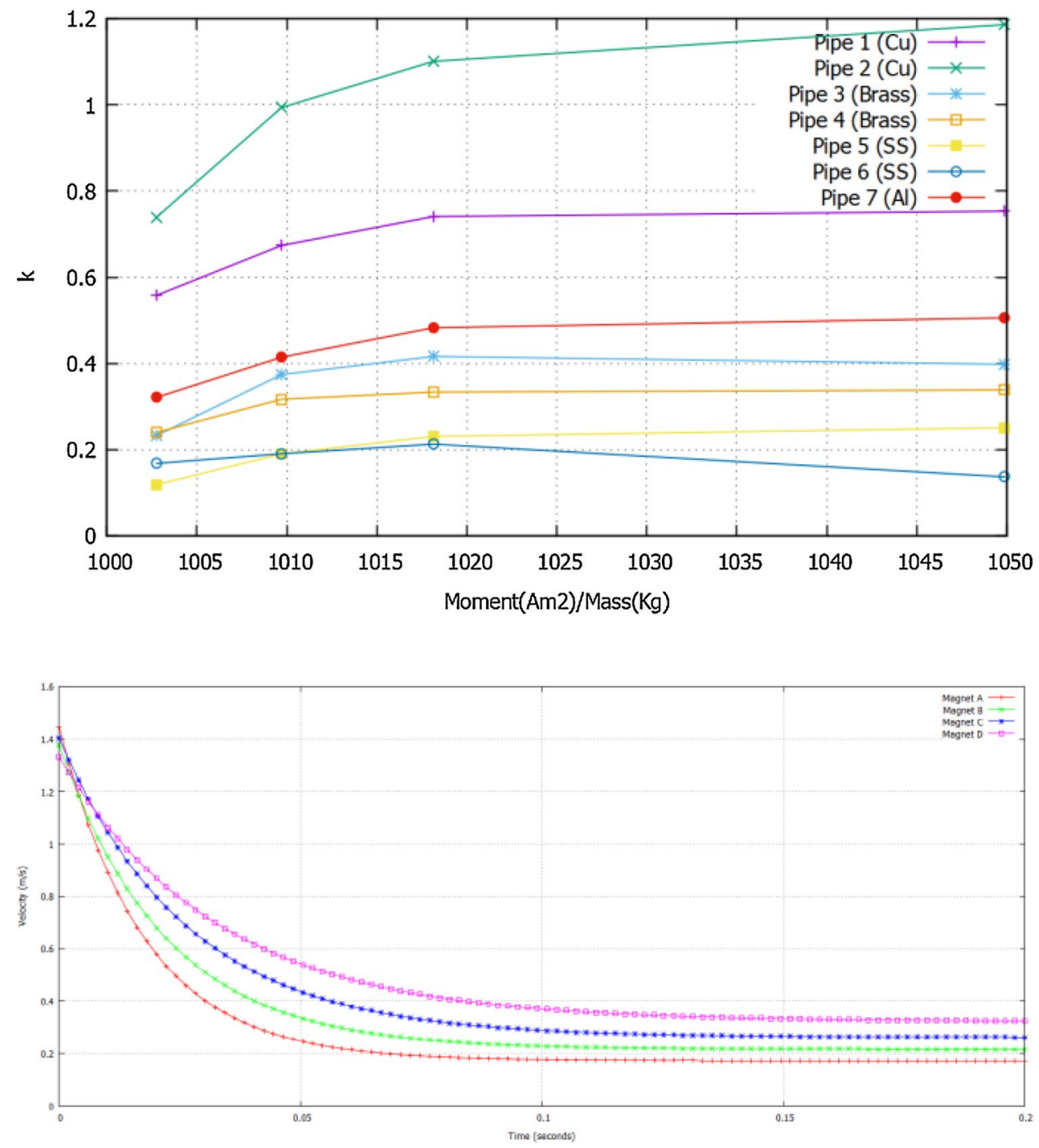
Fig. 8 emf versus time and flux versus time plots for two and four magnets
Table 6 Maximum value of flux for each magnet
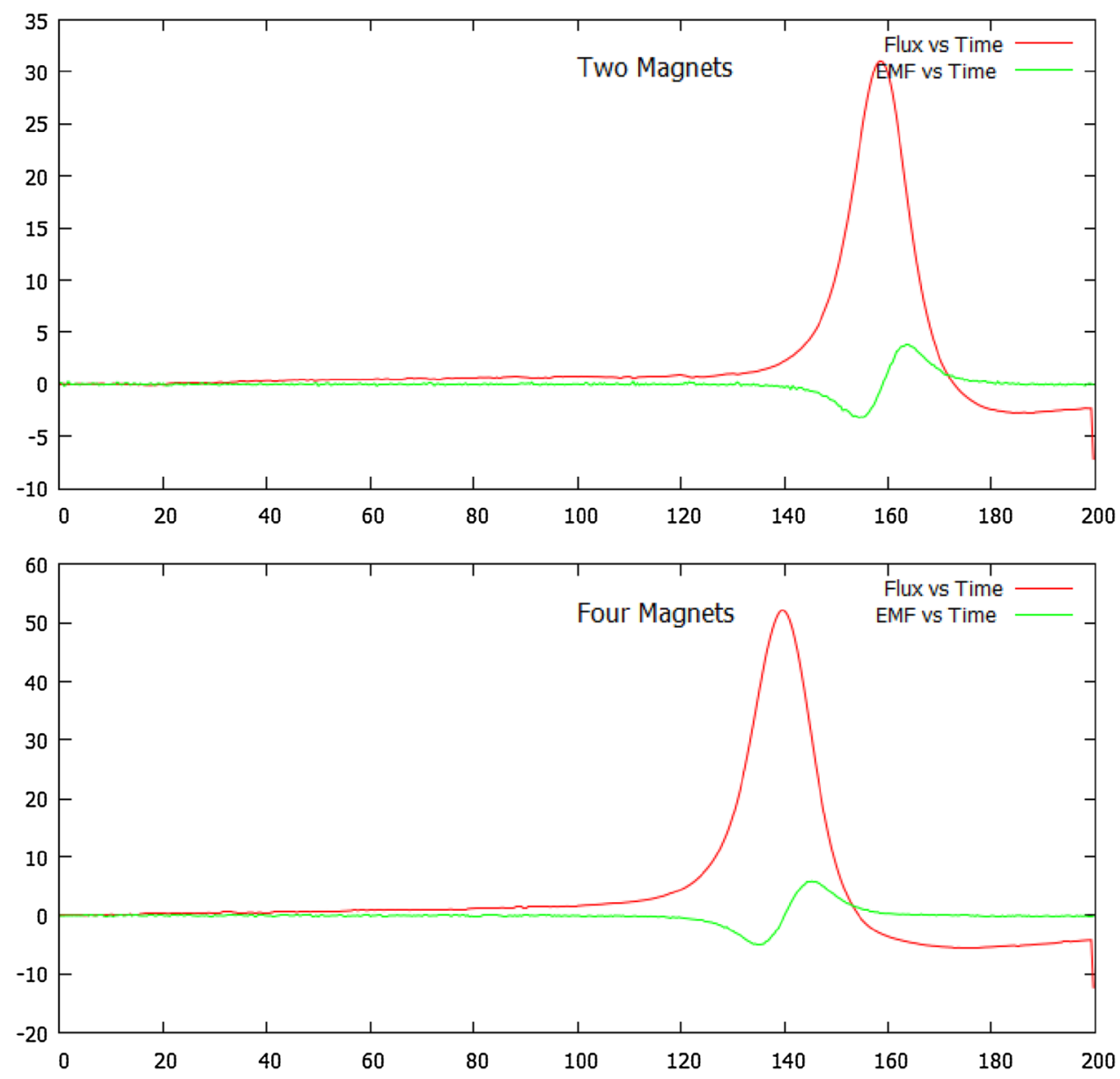

Table 7 Calculated and standard value of conductivity

\begin{tabular}{lll}
\hline Pipe type & $\begin{array}{l}\text { Conductivity calcu- } \\
\text { lated } \times 10^{7}\left(\mathrm{~S} \mathrm{~m}^{-1}\right)\end{array}$ & $\begin{array}{l}\text { Conductivity } \\
\text { standard value } \times 10^{7} \\
\left(\mathrm{~S} \mathrm{~m}^{-1}\right)\end{array}$ \\
\hline $\mathrm{Cu}$ (P1) & 19.696 & 5.96 \\
$\mathrm{Cu}$ (P2) & 23.498 & 5.96 \\
Brass (P3) & 12.908 & 1.69 \\
Brass (P4) & 5.977 & 1.69 \\
SS (P5) & 4.549 & 0.14 \\
SS (P6) & 4.931 & 0.14 \\
Al (P7) & 17.593 & 3.77 \\
\hline
\end{tabular}

\begin{tabular}{ll}
\hline Magnet & Flux $10^{-5}(\mathrm{~Wb})$ \\
\hline A & 3.10218 \\
B & 3.90463 \\
C & 5.21512 \\
D & 6.30849 \\
\hline
\end{tabular}

Acknowledgements We are highly indebted to Dr. Mohamed Shahin Thayyil, Assistant Professor, Department of Physics, University of Calicut, B.P.Ajithkumar, Senior Scientist, IUAC, New Delhi, P.O. Muneera, K. M. Najiya Maryam, M.K. Abdurahiman alias Bava and other Committee Members of Tirurangadi Orphanage and PSMO College for their invaluable support and encouragement. We are very much thankful to the two unknown reviewers who helped us to revise the manuscript into a publishable format. The ExpEYES17 data logger for this work was supplied by Dr. Mohamed Shahin Thayyil, University of Calicut, which was purchased under the project 'RISE' of Dr. Mohamed Shahin Thayyil and Dr. Abdul Kareem Thottoli, financially supported by Kerala State Council for Science, Technology and Environment (KSCSTE), Kerala, India. This paper discusses the work carried out in our lab as a part of graduate degree project work (BSc Physics Project Work). This activity can be performed to enhance the students' knowledge about the properties of magnet and metal pipes. The data were extracted using a data acquisition tool ExpEYES17.

\section{Compliance with ethical standards}

Conflict of interest The authors declare that they have no conflict of interest. 


\section{References}

1. Îniguez J, Zazo M, Flores AG, Hernández-López $A$, Raposo V (2005) Measurement of the electrical conductivity of metallic tubes by studying magnetic screening at low frequency. Am J Phys 73(3):206-210

2. Partovi MH, Morris EJ (2006) Electrodynamics of a magnet moving through a conducting pipe. Can J Phys 84(4):253-271

3. Bae JS, Hwang JH, Park JS, Kwag DG (2010) Modeling and experiments on eddy current damping caused by a permanent magnet in a conductive tube. J Mech Sci Technol 23(11):3024-3035

4. González MI (2004) Experiments with eddy currents: the eddy current brake. Eur J Phys 25(4):463-468

5. Irvine B, Kemnetz M, Gangopadhyaya A, Ruubel T (2014) Magnet traveling through a conducting pipe: a variation on the analytical approach. Am J Phys 82:273

6. Hahn KD, Johnson EM, Brokken A, Baldwin S (2005) Eddy current damping of a magnet moving through a pipe. Am J Phys 66(12):1066-1076

7. Íniguez J, Raposo V, Hernández-López A, Flores AG, Zazo M (2004) Study of the conductivity of a metallic tube by analysing the damped fall of a magnet. Eur J Phys 25(5):593-604

8. Najiya Maryam KM (2014) EM induction experiment to determine the moment of a magnet. Phys Educ 49(3):319-325

9. Donoso G, Ladera CL, Martín P (2011) Damped fall of magnets inside a conducting pipe. Am J Phys 79(2):193-200

10. Donoso G, Ladera CL, Martín P (2009) Magnet fall inside a conductive pipe: motion and the role of the pipe wall thickness. Eur J Phys 30(4):855-869

11. Ruber K, Kanapathipillai S, Randall R (2014) Investigations of eddy current vibration damping. In: 43rd International congress noise control engineering. pp 1-14

12. Dufour S, Vinsard G, Brancher JP (2012) Lateral forces on a magnet falling in a conducting pipe. COMPEL Int J Comput Math Electr Electron Eng 31(6):1625-1636

13. Goff JE (2004) Heuristic model of air drag on a sphere. Phys Educ 39(6):496-499

Publisher's Note Springer Nature remains neutral with regard to jurisdictional claims in published maps and institutional affiliations. 